\title{
The effects of Zn Impurity on the Properties of Doped Cuprates in the Normal State
}

\author{
Yun Song \\ Department of Physics, Beijing Normal University, Beijing 100875, China
}

(November 4, 2018)

\begin{abstract}
We study the interplay of quantum impurity, and collective spinon and holon dynamics in $\mathrm{Zn}$ doped high- $\mathrm{T}_{c}$ cuprates in the normal state. The two-dimensional $t-\mathrm{t}^{\prime}-\mathrm{J}$ models with one and a small amount of $\mathrm{Zn}$ impurity are investigated within a numerical method based on the double-time Green function theory. We study the inhomogeneities of holon density and antiferromagnetic correlation background in cases with different $\mathrm{Zn}$ concentrations, and obtain that doped holes tend to assemble around the $\mathrm{Zn}$ impurity with their mobility being reduced. Therefore a bound state of holon is formed around the nonmagnetic $\mathrm{Zn}$ impurity with the effect helping $\mathrm{Zn}$ to introduce local antiferromagnetism around itself. The incommensurate peaks we obtained in the spin structure factor indicate that $\mathrm{Zn}$ impurities have effects on mixing the $\mathrm{q}=(\pi, \pi)$ and $\mathrm{q}=0$ components in spin excitations.

75.30.Hx, 74.72.-h, 74.20.Mn
\end{abstract}

The effects of divalent transition metal $\mathrm{Zn}$ substitute for $\mathrm{Cu}$ in $\mathrm{CuO}_{2}$ plane present much valuable information in understanding the mechanism of high temperature superconductors. $\mathrm{Zn}^{2+}$ has a closed $d$ shell with spin $s=0$, and acts as a very strong scattering center. As a result, the spin configurations and the electronic structures around the nonmagnetic impurity $\mathrm{Zn}$ are strongly disrupted in both the normal state and superconducting state. Below $T_{c}$, there have been many experimental $[1-5]$ and theoretical [6-10] investigations to discover how the $d$-wave superconductivity is destroyed and what is the microscopic mechanism behind. As the normal state properties are more fundamental, some experimental [11-15] and theoretical [16-18] works have been done to study the effects of nonmagnetic impurity $\mathrm{Zn}$ on the properties of normal state. It is believed that these studies are of great help for understanding the peculiar behaviors of normal state. Moreover, they shed light on the recent striking issue of the normal state pseudogap $[19,20]$. So far, there has been no clear picture of how the impurity interplays with the strong correlation background. It still remains unanswered why the $\mathrm{Zn}$ impurity produced very strong scatter and how the impurity band forms in real space.

In this paper, we perform numerical calculation to study the effects of $\mathrm{Zn}$ impurity on its surrounded $\mathrm{Cu}$ ions in the normal state. We want to find out how the Zn impurity influences the hole distribution and AF correlation background, which may bring about a better understanding of the fundamental relation between spin and hole. We start from the two-dimensional (2D) $t-t^{\prime}-$ $\mathrm{J}$ model and use fermion-spin theory [21]. Fermion-spin theory is based on the charge-spin separation, in which the single occupied constrain of $\mathrm{t}^{-} \mathrm{t}^{\prime}-\mathrm{J}$ model could be treated properly even in the mean field approximation. Within an improved Green function theory [22], we perform numerical calculation for cases with only one $\mathrm{Zn}$ impurity and a small amount of Zn impurity, and the effect of $\mathrm{Zn}$ concentration on some properties of the normal state are discussed.

The essential physics of high- $T_{c}$ cuprates is well described by the t-J model on a square lattice. In the condition that $\mathrm{Zn}$ substitutes $\mathrm{Cu}$ in $\mathrm{CuO}_{2}$ plane, we can model Zn impurity as vacant site, which has no coupling with surrounded $\mathrm{Cu}$ sites. We add the next-nearest-neighbor hopping term in our model to reproduce the realistic band structure, and start our study from the following Hamiltonian

$$
\begin{aligned}
H= & -t \sum_{\langle i, j\rangle \neq l, \sigma}\left(C_{i \sigma}^{\dagger} C_{j \sigma}+\text { h.c. }\right)-t^{\prime} \sum_{\left\langle i, i^{\prime}\right\rangle \neq l, \sigma}\left(C_{i \sigma}^{\dagger} C_{i^{\prime} \sigma}+h . c .\right) \\
& -\mu \sum_{i, \sigma} C_{i \sigma}^{\dagger} C_{i \sigma}+J \sum_{\langle i, j\rangle \neq l} \mathbf{S}_{\mathbf{i}} \cdot \mathbf{S}_{\mathbf{j}}
\end{aligned}
$$

where $\langle i, j\rangle$ and $\left\langle i, i^{\prime}\right\rangle$ mean the summations over nearestneighbor (NN) and next-nearest-neighbor (NNN) pairs respectively, and $l$ represents the site occupied by $\mathrm{Zn}$ impurity. In our model the direct hopping among a Zn impurity and its surrounded $\mathrm{Cu}$ sites is forbidden. In addition, to eliminate the doubly occupied sites of $\mathrm{Cu}$ ion, we introduce constraint $\sum_{\sigma} C_{i \sigma}^{\dagger} C_{i \sigma} \leq 1$ for each $\mathrm{Cu}$ site. Also we introduce $\sum_{\sigma} C_{l \sigma}^{\dagger} C_{l \sigma}=2$ for $\mathrm{Zn}^{2+}$ ion since it has a closed $d$ shell. Therefore, the total number of electrons satisfies $\sum_{i \sigma} C_{i \sigma}^{\dagger} C_{i \sigma}=N-N_{h}+N_{Z n}$ with $N_{h}$ and $N_{Z n}$ representing the number of hole and $\mathrm{Zn}$ impurity, respectively. As the strong electron correlation manifests itself by the local constraint, the key issue is how to treat the constraint properly.

Here we study the $t-\mathrm{t}^{\prime}-\mathrm{J}$ model within the fermion-spin theory [21] based on the charge-spin separation. We introduce $C_{i \uparrow}=h_{i}^{\dagger} S_{i}^{-}$and $C_{i \downarrow}=h_{i}^{\dagger} S_{i}^{+}$, where the spinless fermion operator $h_{i}$ describes the charge (holon) degrees of freedom, while the pseudospin operator $S_{i}$ describes the spin (spinon) degrees of freedom. Thus the low energy behavior of the $\mathrm{t}^{-} \mathrm{t}^{\prime}-\mathrm{J}$ model (1) can be written as

$$
H=-t \sum_{\langle i, j\rangle \neq l}\left(h_{i} h_{j}^{\dagger}+h_{j} h_{i}^{\dagger}\right)\left(S_{i}^{+} S_{j}^{-}+S_{i}^{-} S_{j}^{+}\right)
$$




$$
\begin{aligned}
& -t^{\prime} \sum_{\left\langle i, i^{\prime}\right\rangle \neq l}\left(h_{i} h_{i^{\prime}}^{\dagger}+h_{i^{\prime}} h_{i}^{\dagger}\right)\left(S_{i}^{+} S_{i^{\prime}}^{-}+S_{i}^{-} S_{i^{\prime}}^{+}\right) \\
& +\mu \sum_{i \neq l} h_{i}^{\dagger} h_{i}+\sum_{\langle i, j\rangle \neq l} J_{i, j}^{\mathrm{eff}} \mathbf{S}_{i} \cdot \mathbf{S}_{j},
\end{aligned}
$$

where $J_{i, j}^{\text {eff }}=\left[\left(1-n_{i}^{h}\right)\left(1-n_{j}^{h}\right)-\phi_{i, j}^{2}\right] J$ with $n_{i}^{h}$ representing the hole concentration at site $i$ and $\phi_{i, j}=\left\langle h_{i}^{\dagger} h_{j}\right\rangle$ being the order parameter of holon. Here we also introduce spinon correlation functions $\chi_{i j}=\left\langle S_{i}^{-} S_{j}^{+}\right\rangle$and $\chi_{i j}^{z}=\left\langle S_{i}^{z} S_{j}^{z}\right\rangle$.

We introduce three double-time Green functions

$$
\begin{aligned}
G_{h}\left(i-j, \tau-\tau^{\prime}\right) & =-i \theta\left(\tau-\tau^{\prime}\right)\left\langle\left[h_{i}(\tau) ; h_{j}^{+}\left(\tau^{\prime}\right)\right]\right\rangle \\
& \equiv\left\langle\left\langle h_{i}(\tau) ; h_{j}^{+}\left(\tau^{\prime}\right)\right\rangle\right\rangle \\
D_{s}\left(i-j, \tau-\tau^{\prime}\right) & =-i \theta\left(\tau-\tau^{\prime}\right)\left\langle\left[S_{i}^{+} ; S_{j}^{-}\left(\tau^{\prime}\right)\right]\right\rangle \\
& \equiv\left\langle\left\langle S_{i}^{+}(\tau) ; S_{j}^{-}\left(\tau^{\prime}\right)\right\rangle\right\rangle \\
D_{s}^{z}\left(i-j, \tau-\tau^{\prime}\right) & =-i \theta\left(\tau-\tau^{\prime}\right)\left\langle\left[S_{i}^{z} ; S_{j}^{z}\left(\tau^{\prime}\right)\right]\right\rangle \\
& \equiv\left\langle\left\langle S_{i}^{z}(\tau) ; S_{j}^{z}\left(\tau^{\prime}\right)\right\rangle\right\rangle,
\end{aligned}
$$

where $G_{h}$ describes the behaviors of holon, and $D_{s}$ and $D_{s}^{z}$ describe the behaviors of spinon. Since the lattice translational invariance is not presented in cases with $\mathrm{Zn}$ impurities, we evaluate the equations of motion of the above Green's functions in real space. The double-time Green function $\langle\langle A ; B\rangle\rangle$ satisfies

$$
\omega\langle\langle A ; B\rangle\rangle_{\omega}=\left\langle[A, B]_{\mp}\right\rangle_{\omega}+\langle\langle[A, H] ; B\rangle\rangle_{\omega},
$$

thus we could obtain the equations of motion of $G_{h}$

$$
\begin{array}{r}
(\omega-\mu) G_{h}(i-j)_{\omega}-2 t \sum_{\eta} \chi_{i, i+\eta} G_{h}(i+\eta-j)_{\omega} \\
-2 t^{\prime} \sum_{\tau} \chi_{i, i+\tau} G_{h}(i+\tau-j)_{\omega}=\delta(i-j) .
\end{array}
$$

We introduce $\widetilde{G}_{h}$, a $N^{2} \times N^{2}$ elements matrix, to express the holon Green functions for a square lattice with $N \times N$ sites. And we could rewrite Eq. (5) as

$$
(\omega-\mu) \widetilde{G_{h}}-\widetilde{h} \widetilde{G_{h}}=\widetilde{I},
$$

where matrix $\widetilde{h}$ is decided by the NN and NNN spinon correlation functions, and $\widetilde{I}$ is an identity matrix.

Based on Eq. (3) and (4), we also obtain the equations of motion of spinon Green functions $D_{s}$ and $D_{s}^{z}$

$$
\begin{aligned}
\omega D_{s}(i-j)_{\omega}= & 2 \sum_{\eta} J_{i, i+\eta}^{e f f}\left\{\epsilon_{i, i+\eta} F_{1}(i, i+\eta ; j)_{\omega}\right. \\
& \left.-F_{1}(i+\eta, i ; j)_{\omega}\right\} \\
& +8 t^{\prime} \sum_{\tau} \phi_{i, i+\tau} F_{1}(i, i+\tau ; j)_{\omega} \\
\omega D_{s}^{z}(i-j)_{\omega}= & \sum_{\eta} J_{i, i+\eta}^{e f f} \epsilon_{i, i+\eta}\left\{F_{2}(i, i+\eta ; j)_{\omega}\right. \\
& \left.-F_{2}(i+\eta, i ; j)_{\omega}\right\} \\
& +4 t^{\prime} \sum_{\tau} \phi_{i, i+\tau}\left\{F_{2}(i, i+\tau ; j)_{\omega}\right. \\
& \left.-F_{2}(i+\tau, i ; j)_{\omega}\right\},
\end{aligned}
$$

where $\epsilon_{i, i+\eta}=1+\frac{4 t \phi_{i, i+\eta}}{J_{i, i+\eta}^{e f f}} . \quad F_{1}$ and $F_{2}$ are the secondorder spinon Green functions which are defined as

$$
\begin{aligned}
& F_{1}(i, l ; j)_{\omega}=\left\langle\left\langle S_{i}^{z} S_{l}^{+} ; S_{j}^{-}\right\rangle\right\rangle_{\omega} \\
& F_{2}(i, l ; j)_{\omega}=\left\langle\left\langle S_{i}^{+} S_{l}^{-} ; S_{j}^{z}\right\rangle\right\rangle_{\omega} .
\end{aligned}
$$

Going a step further, we establish the equations of motion of the second-order spinon Green functions

$$
\begin{aligned}
\omega F_{1}(i, l ; j)_{\omega}= & 2 \chi_{i, l}^{z} \delta(l-j)-\chi_{i, l} \delta(i-j) \\
+ & \left\langle\left\langle\left\{\sum _ { \eta } \left[ 2 J_{l, l+\eta}^{e f f}\left(\epsilon_{l, l+\eta} S_{i}^{z} S_{l}^{z} S_{l+\eta}^{+}-S_{i}^{z} S_{l+\eta}^{z} S_{l}^{+}\right)\right.\right.\right.\right. \\
& \left.+J_{i, i+\eta}^{e f f} \epsilon_{i, i+\eta}\left(S_{i}^{+} S_{i+\eta}^{-} S_{l}^{+}-S_{i+\eta}^{+} S_{i}^{-} S_{l}^{+}\right)\right] \\
& +4 t^{\prime} \sum_{\tau}\left[\phi_{i, i+\tau}\left(S_{i}^{+} S_{i+\tau}^{-} S_{l}^{+}-S_{i+\tau}^{+} S_{i}^{-} S_{l}^{+}\right)\right. \\
+ & \left.\left.\left.\left.2 \phi_{l, l+\tau} S_{i}^{z} S_{l}^{z} S_{l+\tau}^{+}\right]\right\} ; S_{j}^{-}\right\rangle\right\rangle_{\omega} \\
\omega F_{2}(i, l ; j)_{\omega}= & \chi_{i, l} \delta(l-j)-\chi_{i, l} \delta(i-j) \\
+ & \left\langle\left\langle\left\{\sum _ { \eta } \left[ 2 J_{l, l+\eta}^{e f f}\left(S_{i}^{+} S_{l}^{-} S_{l+\eta}^{z}-\epsilon_{l, l+\eta} S_{i}^{+} S_{l+\eta}^{-} S_{l}^{z}\right)\right.\right.\right.\right. \\
+ & \left.2 J_{i, i+\eta}^{e f f}\left(\epsilon_{i, i+\eta} S_{i+\eta}^{+} S_{l}^{-} S_{i}^{z}-S_{i}^{+} S_{l}^{-} S_{i+\eta}^{z}\right)\right] \\
+ & 8 t^{\prime} \sum_{\tau}\left[\phi_{i, i+\tau} S_{i+\tau}^{+} S_{l}^{-} S_{i}^{z}\right. \\
& \left.\left.\left.\left.-\phi_{l, l+\tau} S_{i}^{+} S_{l+\tau}^{-} S_{l}^{z}\right]\right\} ; S_{j}^{z}\right\rangle\right\rangle_{\omega} .
\end{aligned}
$$

To the third-order spinon Green functions in the right hand side of Eq. (9), we perform the improved decoupling scheme as described in Ref. 22, for example

$$
\left\langle\left\langle S_{i}^{z} S_{l}^{z} S_{l+\eta}^{+} ; S_{j}^{-}\right\rangle\right\rangle \rightarrow \alpha_{i}\left\langle S_{i}^{z} S_{l}^{z}\right\rangle \alpha_{l}\left\langle\left\langle S_{l+\eta}^{+} ; S_{j}^{-}\right\rangle\right\rangle .
$$

Therefore, the second-order spinon Green functions $F_{1}$ and $F_{2}$ can be expressed by the Green functions $D_{s}$ and $D_{s}^{z}$ as

$$
\begin{aligned}
\omega F_{1}(i, l ; j)_{\omega} & =\Gamma_{1}^{0}+\Gamma_{1}^{1} D(i-j)+\Gamma_{1}^{4} D(l-j) \\
& +\sum_{\eta}\left\{\Gamma_{1}^{2} D(i+\eta-j)+\Gamma_{1}^{3} D(l+\eta-j)\right\} \\
& +\sum_{\tau}\left\{\Gamma_{1}^{5} D(i+\tau-j)+\Gamma_{1}^{6} D(l+\tau-j)\right\} \\
\omega F_{2}(i, l ; j)_{\omega} & =\Gamma_{2}^{0}+\Gamma_{2}^{1} D^{z}(i-j)+\Gamma_{2}^{4} D^{z}(l-j) \\
& +\sum_{\eta}\left\{\Gamma_{2}^{2} D^{z}(i+\eta-j)+\Gamma_{2}^{3} D^{z}(l+\eta-j)\right\},
\end{aligned}
$$

where

$$
\begin{aligned}
\Gamma_{1}^{0}= & 2 \chi_{i, l}^{z} \delta(l-j)-\chi_{i, l} \delta(i-j) \\
\Gamma_{1}^{1}= & \sum_{\eta} J_{i, i+\eta}^{e f f} \epsilon_{i, i+\eta} \alpha_{i+\eta} \chi_{i+\eta, l} \alpha_{l} \\
& +4 t^{\prime} \sum_{\tau} \phi_{i, i+\tau} \alpha_{i+\tau} \chi_{i+\tau, l} \alpha_{l} \\
\Gamma_{1}^{2}= & -J_{i, i+\eta}^{e f f} \epsilon_{i, i+\eta} \alpha_{i} \chi_{i, l} \alpha_{l}
\end{aligned}
$$




$$
\begin{aligned}
\Gamma_{1}^{3}= & 2 J_{l, l+\eta}^{e f f} \epsilon_{l, l+\eta} \alpha_{i} \chi_{i, l}^{z} \alpha_{l} \\
\Gamma_{1}^{4}= & -2 \sum_{\eta} J_{l, l+\eta}^{e f f} \alpha_{i} \chi_{i, l+\eta}^{z} \alpha_{l+\eta} \\
\Gamma_{1}^{5}= & -4 t^{\prime} \phi_{i, i+\tau} \alpha_{i} \chi_{i, l} \alpha_{l} \\
\Gamma_{1}^{6}= & 8 t^{\prime} \phi_{l, l+\tau} \alpha_{i} \chi_{i, l}^{z} \alpha_{l} \\
\Gamma_{2}^{0}= & \chi_{i, l} \delta(l-j)-\chi_{i, l} \delta(i-j) \\
\Gamma_{2}^{1}= & 2 \sum_{\eta} J_{i, i+\eta}^{e f f} \epsilon_{i, i+\eta} \beta_{i+\eta} \chi_{i+\eta, l} \beta_{l} \\
& +8 t^{\prime} \sum_{\tau} \phi_{i, i+\tau} \beta_{i+\tau} \chi_{i+\tau, l} \beta_{l} \\
\Gamma_{2}^{2}= & -2 J_{i, i+\eta}^{e f f} \beta_{i} \chi_{i, l} \beta_{l} \\
\Gamma_{2}^{3}= & 2 J_{l, l+\eta}^{e f f} \beta_{i} \chi_{i, l} \beta_{l}
\end{aligned}
$$

and

$$
\begin{aligned}
\Gamma_{2}^{4}= & -2 \sum_{\eta} J_{l, l+\eta}^{e f f} \epsilon_{l, l+\eta} \beta_{i} \chi_{i, l+\eta} \beta_{l+\eta} \\
& -8 t^{\prime} \sum_{\tau} \phi_{l, l+\tau} \beta_{i} \chi_{i, l+\tau} \beta_{l+\tau} .
\end{aligned}
$$

We also introduce two $N^{2} \times N^{2}$ elements matrices $\widetilde{D}_{s}$ and $\widetilde{D}_{s}^{z}$ to express the spion Green functions. Based on Eq. (7) and (11), we could obtain that

$$
\begin{aligned}
\omega^{2} \widetilde{D_{s}}-\widetilde{S} \widetilde{D_{s}} & =\widetilde{C_{s}} \\
\omega^{2} \widetilde{D_{s}^{z}}-\widetilde{S^{z}} \widetilde{D_{s}^{z}} & =\widetilde{C_{s}^{z}},
\end{aligned}
$$

where matrices $\widetilde{S}, \widetilde{S^{z}}, \widetilde{C_{s}}$ and $\widetilde{C_{s}^{z}}$ are decided by the density of holon $n_{i}$, and the spin correlation functions.

We establish the self-consistent equations based on Eq. (6) and (12) to determine the correlation functions of holon and spinon, and also the vertex correction parameters. Under the periodic boundary conditions, we have performed numerical calculations for $16 \times 16$ and $20 \times 20$ lattices with different Zn concentrations respectively. Based on the experimental results of BSCCO near optimal doping $[1,9]$, the parameters of $\mathrm{t}-\mathrm{t}^{\prime}-\mathrm{J}$ model are taken as $t / J=2.5$ and $t^{\prime} / t=-0.4$ in our calculations.

Firstly, we study the $20 \times 20$ lattice with only one $\mathrm{Zn}$ impurity in the optimally doped region $\left(\delta_{h}=0.15\right)$. The spacial distribution of holon density is calculated and our numerical results are shown in figure 1 . We find that the holon density closet to the Zn impurity oscillates strongly, and the fluctuation diminishes rapidly away from the $\mathrm{Zn}$ impurity. The maximum density is obtained at sites two lattice distances away from the $\mathrm{Zn}$ impurity, and is about $15 \%$ higher than the minimum density. Our numerical results suggest that doped holes form a local region around the $\mathrm{Zn}$ impurity, whose size is about eight lattice cells as shown in figure 1 . We also obtain that the magnetic modification introduced by $\mathrm{Zn}$ is mainly in the vicinity of $\mathrm{Zn}$ impurity. The NN correlation functions near the isolated nonmagnetic impurity in the undoped case have been discussed carefully in Ref. 22. We have found that Zn impurity strongly modifies the spin excitations, especially the magnetic properties of the neighbor $\mathrm{Cu}$. As a result, the $\mathrm{AF}$ correlation functions at the bonds closed to the impurities are enhanced. In the optimally doped region, we also obtain that $\mathrm{Zn}$ impurity enhances the $\mathrm{NN}$ AF correlation functions of spinons close to it. Moreover, The doped holes have the effect to strengthen the AF correlations near the Zn impurity. The ${ }^{63} \mathrm{Cu}$ NMR study of $\mathrm{YBa}_{2}\left(\mathrm{Cu}_{0.99} \mathrm{Zn}_{0.01}\right)_{3} \mathrm{O}_{6.7}$ also find that the $\mathrm{AF}$ correlations are enhanced, not destroyed, around Zn impurities [13]. Since the quantum fluctuation of spinons close to the nonmagnetic impurity is reduced obviously, we can divided the system into strong AF correlation region and weak AF correlation region. The tendency of doped holes to assemble around the Zn impurity could rationalize anomalous charge localization effect, and the mobility of those holons closed to the $\mathrm{Zn}$ impurity could also be reduced. Therefore, a bound state of holon [23] is formed around the $\mathrm{Zn}$ impurity. Bound state of impurity in normal state has also been predicted by the self-consistent $T$ matrix approach [18].

We also study the cases with several Zn impurities in the optimally doped regime, and find that the holes play different roles in the strong AF correlation region and the weak AF correlation region. Our numerical results show that the AF correlations of bonds far from the $\mathrm{Zn}$ impurity reduces remarkably as hole is added into the systems. On the contrary, the bound state of holon has the effect to enhance the AF correlations around Zn impurity, and helps $\mathrm{Zn}$ to introduce local antiferromagnetism around itself. As $\mathrm{Zn}$ concentration increases, the $\mathrm{CuO}_{2}$ plane becomes a inhomogeneous mixture of strong AF correlation regions and weak AF correlation regions. And the doped holes tend to assemble at the strong AF correlation regions.

We show the density of state of holon in cases with several Zn impurities in figure 2. Jung et al [24] have examined some samples and prove that $\mathrm{Zn}$ is uniformly distributed. In some configurations when $\mathrm{Zn}$ is uniformly distributed, our calculation show that due to the strong coupling between impurity and the conduction band, the width of the holon band decreases as $\mathrm{Zn}$ concentration increases. We find that the inhomogeneity of the spinon background increases the density of state of holon near the fermi surface, and a resonance peak of impurity states is found to get broader and stronger as $\mathrm{Zn}$ concentration increases. Inelastic neutron scattering study for the optimal doped $\mathrm{La}_{1.85} \mathrm{Sr}_{0.15} \mathrm{Cu}_{1-y} \mathrm{Zn}_{y} \mathrm{O}_{4}$ indicates that a new in-gap Zn impuriy state is introduced at low temperature [25]. Nonmagnetic defect structures at the surface has also been found to create localized low-energy excitations in their immediate vicinity in $\mathrm{Bi}_{2} \mathrm{Sr}_{2} \mathrm{CaCu}_{2} \mathrm{O}_{8}$ by performing low-temperature tunneling spectroscopy measurements with a scanning tunneling microscope [4]. 
Our calculations show that the impurity state can survive above $T_{c}$, which is in agreement with the theoretical prediction [18] as well.

To study the effect of the $\mathrm{Zn}$ impurity on the spin background around it, we introduce the spin structure factor

$$
\mathbf{S}_{i}(\mathbf{k})=\sum_{j} S_{i}^{z} S_{j}^{z} e^{\mathbf{k} \cdot(\mathbf{i}-\mathbf{j})} .
$$

Here $i$ represents $\mathrm{Cu}$ site around $\mathrm{Zn}$ impurity. Zn impurity is a scatter which has a strong effect on the AF correlation background [26]. As Zn concentration increases, the $\mathrm{CuO}_{2}$ plane becomes a inhomogeneous mixture of strong AF correlation regions and weak AF correlation regions. In figure 3 , the numerical results of the $\mathrm{NN} \mathrm{Cu}$ sites around $\mathrm{Zn}$ impurity are shown for $16 \times 16$ lattices with different $\mathrm{Zn}$ concentrations. We obtain that, in the pure case and Zn lightly doped case $\left(\delta_{Z n} \leq 0.01\right)$, the spin excitations are dominated by a magnetic resonance peak located at $Q_{A F}=(\pi, \pi)$. As the $\mathrm{Zn}$ concentration increases, this peak decreases and there appear two second-high incommensurate peaks as shown in figure 3, which results from the mixing of $q=(\pi, \pi), q=(\pi, 0)$ and $q=0$ components in spin excitations introduced by the strong impurity scattering. In Bulut's study of susceptibility of $\mathrm{Zn}$ doped high- $T_{c}$ superconductors, the similar behaviors are also obtained [17]. In addition, we find that the distances of the incommensurate peaks from $q=(\pi, \pi)$ increase with doping, and these peaks become broad and weak in amplitude with the increasing of $\mathrm{Zn}$ concentration. Meanwhile, as a result of the increasing of the disorder introduced by the $\mathrm{Zn}$ substitution on the $\mathrm{Cu}$ sites, the peak at $q=(\pi, \pi)$ decreases gradually as the $\mathrm{Zn}$ concentration increases, and disappears when $\delta_{Z n} \geq 0.1$. Thus the result is consistent with experimental results of Zn-doped high- $T_{c}$ cuprates [11].

In summary, we have studied the interplay between quantum impurities, and collective spinon and holon dynamics in Zn-doped cuprate in the normal state. Within a numerical method based on the Green function theory, the inhomogeneities of holon density distribution and antiferromagnetic correlation background in twodimensional $\mathrm{t}-\mathrm{t}^{\prime}-\mathrm{J}$ model with Zn impurities are investigated. We obtain the real space shape of bound state of holon surrounding the nonmagnetic Zn impurity. We also find that the doped holes help $\mathrm{Zn}$ to introduce local antiferromagnetism around itself. In the cases with a small amount of $\mathrm{Zn}$ impurities, the influence of $\mathrm{Zn}$ impurity on the antiferromagnetic correlation background is studied. The appearance of incommensurate peaks in spin structure factor indicates that $\mathrm{Zn}$ impurity is a strong scatter center, which has an effect on mixing the $q=(\pi, \pi)$, $q=(\pi, 0)$ and $q=0$ components in spin excitations.

\section{ACKNOWLEDGMENTS}

The authors would like to thank Prof. Feng for helpful discussions. This work was supported by the Grant from Beijing Normal University.

[1] S. H. Pan, E. W. Hudson, K. M. Lang, H. Eisaki, S. Uchida, and J. C. Davis, Nature 403746 (2000).

[2] Y. Sidis, P. Bourges, H. F. Fong, B. Keimer, L. P. Regnault, J. Bossy, A. Ivanov, B. Hennion, P. GautierPicard, G. Collin, D. L. Millius, and I. A. Aksay, Phys. Rev. Lett. 84, 5900 (2000).

[3] E. W. Hudson, S. H. Pan, A. K. Gupta, K. -W. Ng, and J. C. Davis, Science 285, 88 (1999).

[4] Ali Yazdani, C. M. Howald, C. P. Lutz, A. Kapitulnik, and D. M. Eigler, Phys. Rev. Lett. 83, 176 (1999).

[5] A. V. Mahajan, H. Alloul, G. Collin, and J. F. Marucco, Phys. Rev. Lett. 72, 3100 (1994).

[6] A. Polkovnikov, S. Sachdev, and M. Vojta, Phys. Rev. Lett. 86, 296 (2001).

[7] A. V. Balatsky, Nature 403717 (2000); M. I. Salkola, A. V. Balatsky, and D. J. Scalapino, Phys. Rev. Lett. 77, 1841 (1996); A. V. Balatsky, M. I. Salkola, and A. Rosengren, Phys. Rev. B 51, 15547 (1995).

[8] Jian-Xin Zhu, T. K. Lee, C. S. Ting, and Chia-Ren Hu, Phys. Rev. B 61, 8667 (2000).

[9] H. Tsuchiura1, Y. Tanaka, M. Ogata, and S. Kashiwaya, Phys. Rev. Lett. 84, 3165 (2000); J. Phys. Soc. Jpn. 68, 2510 (1999).

[10] T. Xiang, Y. H. Su, C. Panagopoulos, Z. B. Su, and L. Yu, Phys. Rev. B 66, 174504 (2002).

[11] H. Alloul, P. Mendels, H. Casalta, J. F. Marucco, and J. Arabski, Phys. Rev. Lett 67, 3140 (1991).

[12] J. Bobroff, H. Alloul, Y. Yoshinari, A. Keren, P. Mendels, N. Blanchard, G. Collin, and J.-F. Marucco, Phys. Rev. Lett. 79, 2117 (1997); J. Bobroff, W. A. MacFarlane, H. Alloul, P. Mendels, N. Blanchard, G. Collin, and J.-F. Marucco, Phys. Rev. Lett. 83, 4381 (1999).

[13] M.-H. Julien, T. Fehér, M. Horvatic, C. Berthier, O. N. Bakharev, P. Ségransan, G. Collin, and J.-F. Marucco, Phys. Rev. Lett. 84, 3422 (2000).

[14] W. A. MacFarlane, J. Bobroff, H. Alloul, P. Mendels, N. Blanchard, G. Collin, and J.-F. Marucco, Phys. Rev. Lett. 85, 1108 (2000).

[15] H. Yamagata, H. Miyamoto, K. Nakamura, M. Matsumura, and Y. Itoh, cond-mat/0304477.

[16] N. Nagaosa and P. A. Lee, Phys. Rev. Lett. 79, 3755 (1997).

[17] N. Bulut, Phys. Rev. B 61, 9051 (2000).

[18] H. V. Kruis, I. Martin, and A. V. Balatsky, Phys. Rev. B 64, 054501 (2001).

[19] J. L. Tallon, C. Bernhard, G. V. M. Williams, and J. W. Loram, Phys. Rev. Lett. 79, 5294 (1997); J. L. Tallon, Phys. Rev. B 58, 5956 (1998).

[20] B. Chattopadhyay, B. Bandyopadhyay, A. Poddar, P. 
Mandal, A. N. Das, and B. Ghosh, Physica C 331, 38 (2000).

[21] Shiping Feng, Z. B. Su, and L. Yu, Phys. Rev. B 49, 2368 (1994); Mod. Phys. Lett. B 7, 1013 (1993); Shiping Feng and Yun Song, Phys. Rev. B 55642 (1997).

[22] Yun Song, H. Q. Lin and Jue-Lian Shen, Phys. Rev. B 58, 9166 (1998); Yun Song, H. Q. Lin and A. W. Sandvik, J. Phys.: Condens. Matter 12, 5275 (2000).

[23] D. Poilblanc, D. J. Scalapino, and W. Hanke, Phys. Rev. Lett. 72, 884 (1994); W. Ziegler, D. Poilblanc, R. Preuss, W. Hanke, and D. J. Scalapino, Phys. Rev. B 53, 8704 (1996).

[24] C. U. Jung, J. Y. Kim, Min-Seok Park, Mun-Seog Kim, Heon-Jung Kim, S. Y. Lee, and Sung-Ik Lee, Phys. Rev. B 65, 172501 (2002).

[25] H. Kimura, M. Kofu, Y. Matsumoto, and K. Hirota, cond-mat/0209428.

[26] O. P. Vajk, P. K. Mang, M. Greven, P. M. Gehring, and J. W. Lynn, Science, 295, 1691 (2002).

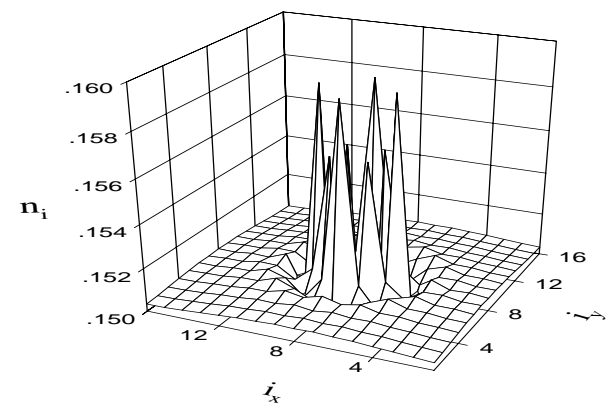

FIG. 1. The distribution of holon density in a $20 \times 20$ lattice with only one Zn impurity.

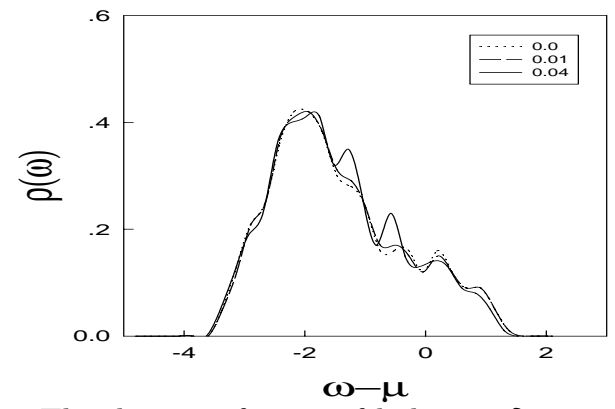

FIG. 2. The density of state of holon at $\delta_{Z n}=0.0$ (dotted line), 0.01(dashed line), and 0.04 (solid line).

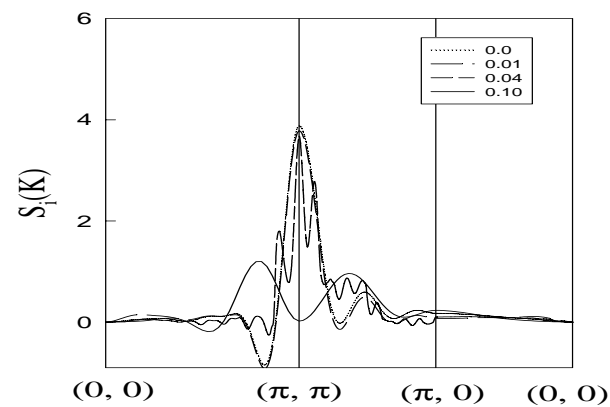

FIG. 3. The Spin structure factor of the nearest neighbor $\mathrm{Cu}$ sites around $\mathrm{Zn}$ impurity for $16 \times 16$ lattices with different Zn concentrations. 